\title{
Leseliste Top 5: Rolle des Controllers
}

In diesem Teil möchten wir Ihnen regelmäßig fünf besonders lesenswerte Bücher zu einem bestimmten Themengebiet vorstellen:

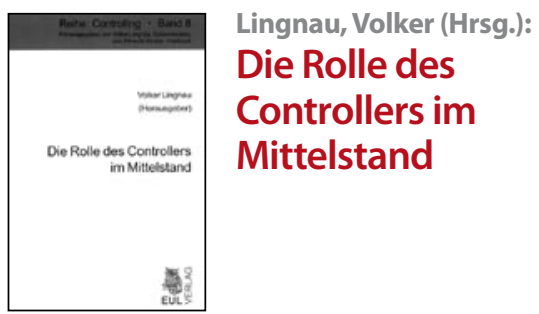

Eul Verlag, Lohmar 2008, 291 S., ISBN: 978-3-89936-688-4, 52,00€

Der 8. Band der Schriftenreihe Controlling beschäftigt sich einleitend mit der Fragestellung eines externen Controllings für Kleinst- und Kleinunternehmen. Anschließend werden die spezifischen Bedürfnisse des Controllings im Mittelstand fokussiert. Dabei wird insbesondere die Rolle des Controllers, das Selbstcontrolling im Projektmanagement als auch die Risikothematik in KMU analysiert. Mit einem Blick auf die Controllinginstrumente im chinesischen Mittelstand finden darüber hinaus internationale Aspekte Berücksichtigung. Ergänzt werden die Beiträge jeweils durch umfangreiche Praxisbeispiele.

Tipp: Die Interaktion von Autoren aus Wissenschaft und Praxis mit dem Anspruch eines ganzheitlichen Blicks auf das Controlling im Mittelstand adressiert eine sehr breite Leserschaft.

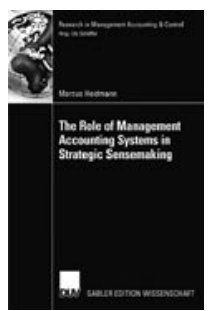

Heidmann, Marcus:

The Role of Management Accounting Systems in Strategic Sensemaking

Gabler Verlag, Wiesbaden 2008, 229 S., ISBN: 978-3-8350-0633-1, 49,90€

Das Buch von Heidmann analysiert die Funktion von Controllingsystemen innerhalb der Strategieentwicklung von Unternehmen. Aufbauend auf der Lerntheorie werden organisationale Veränderungen, Strategiefindungsprozesse und Management Accounting Systeme zunächst theo- retisch fundiert. Im Anschluss daran wird eine Fallstudie vorgestellt, in welcher der Autor Unternehmen aus Luft- und Raumfahrt-, Automobil-sowie Informations- und Kommunikationsindustrie untersucht. Das Resultat verdeutlicht, dass sowohl Management Accounting Systeme als auch informelle Quellen einen Einfluss auf den Strategieentwicklungsprozess haben.

Tipp: In diesem Buch wird die Rolle von Management Accounting Systemen auf die strategische Ausrichtung aus wissenschaftlicher und praktischer Sicht herausgearbeitet. Somit liefert es ein vielfältiges Transferpotenzial für die Unternehmenspraxis.

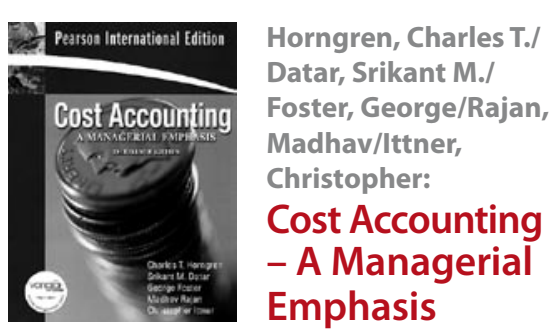

Pearson Education, New Jersey 2008, 895 S., ISBN: 978-0-13-135558-3, 73,95€

Gegenstand dieses Buches ist die umfangreiche Einführung in die Grundlagen des Controllings. Anhand vielfältiger Beispiele aus der Praxis erläutern hochkarätige Autoren die wichtigsten Controllinginstrumente in anschaulicher Art und Weise. Die Bandbreite des Buches reicht dabei von Activity-Based Costing über Budgetplanung sowie Balanced Scorecard bis hin zu Performance Measurement Systemen. Zahlreiche Grafiken und Beispielrechnungen erhöhen die Verständlichkeit für den Leser.

Tipp: Dieses Buch richtet sich aufgrund seiner Vielseitigkeit sowohl an Studenten und Dozenten als auch an Unternehmensvertreter.

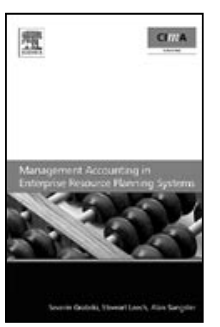

Grabski, Severin/Leech, Stewart/Sangster, Alan Management Accounting in Enterprise Resource Planning Systems

CIMA Publishing, Oxford 2009, 150 S., ISBN: 978-1-85617-679-8, 47,00€
In diesem Buch befassen sich die Autoren mit der Veränderung der Aufgaben und des Verhaltens von Management Accountants aufgrund der Einführung eines ERPSystems. Die Analyse basiert auf Fallstudien in sieben Unternehmen mit jeweils unterschiedlichen Erfolgsgraden bei der Implementierung eines solchen Systems. Die Resultate geben somit insbesondere Hinweise auf Erfolgsfaktoren bei der Einführung im eigenen Unternehmen. Wesentliche Erkenntnisse sind einerseits die Veränderung der Rolle der Controller vom Informationslieferanten hin zum Business Partner; andererseits kann sowohl eine erhebliche Verbesserung der Daten- als auch der Entscheidungsqualität beobachtet werden.

Tipp: Angesichts wertvoller Hilfestellung bei der Implementierung von ERP-Systemen richtet sich dieses Buch insbesondere an Unternehmensvertreter aus Management und Controlling.

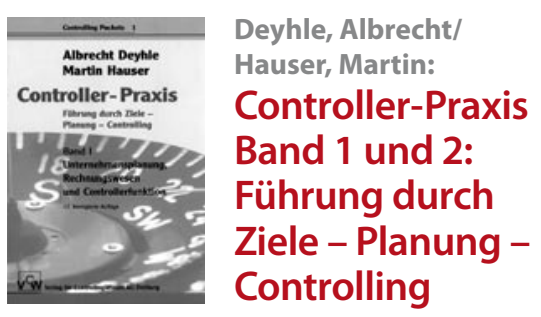

VCW Verlag, Freiburg 2010, Bd. 1: 214 S.; Bd. 2: 217 S., ISBN: 978-3-7775-0037-9, 39,00€

Der inhaltliche Fokus des ersten Bandes liegt auf den Themen Unternehmensplanung, Rechnungswesen und Controllerfunktion. Dabei bilden das Leitbild und die Zielsetzung der Unternehmung sowie die strategische als auch operative Unternehmensplanung den Mittelpunkt der Ausführungen. Letztere wird anhand einer praxisrelevanten Fallstudie anschaulich beleuchtet. Zu den Schwerpunkten des zweiten Bandes gehören Soll-ist-Vergleiche, Erwartungsrechnungen sowie das Thema Führungsstil. Hier wird insbesondere das Controller-Leitbild und die Relevanz der Kommunikationsfähigkeit der Controller in den Vordergrund gerückt.

Tipp: Wie schon der Titel vermuten lässt, beinhalten beide Bände umfangreiche Tipps für eine praxisorientierte Leserschaft. 